\title{
insubstantial stuff of pure being
}

Unbeknownst to me, a door opens under my bed. For a moment there is a transference, as though bound trains puff the sky. I know from the drip marks all about men's viscosity. Just think, I might have been a clarinet with all that wandering. Black instruments. No one has properly named desire, except by what is untasted, even festering. There-with this giant girl, no acts of dominion. A smokehouse, this city, making lost sponges indivisible to touch. The sky holds unsleeping, tired out angels. A terrible insult is this skirt, clotted in gold. 